\title{
Structural means of assessing the association of Hamartin and Tuberin
}

\author{
Nalini Natarajan ${ }^{1}$, Vijay Thiruvenkatam ${ }^{1}$ \\ ${ }^{1}$ Indian Institute Of Technology, Gandhinagar, Gandhinagar, India \\ E-mail: nalini.natarajan@iitgn.ac.in
}

mTOR pathway is one of the most important and well-studied signalling cascades with respect to cancer. The major controllers of mTOR are the tumour suppressors Hamartin (TSC1) and Tuberin (TSC2), which lies in the heart of this cellular signalling pathway. Mutation in any of these genes will render them unsuccessful to possess tuberin's GTPase activity towards Rheb, a small G-protein, which eventually keeps the mTOR activated. Such mutations lead to tuberous sclerosis complex, a multi-organ disease characterized by non-cancerous tumours in brain, skin, liver, kidney, lungs and heart. Also, it puts an individual at a higher risk of developing cancer in these organs.

Several inhibitors are designed and still being designed for mTOR, while the actual source of the problem (TSC1 \& TSC2) is left unchecked. The main reason for this is the lack of structure for both TSC1 and TSC2. Owing to its large size, Hamartin (1164 aa) and Tuberin (1804 aa) cannot be expressed under a bacterial expression system. Hence the major focus of our study is in the interaction regions of these two proteins. Since it is a small portion of these proteins, its expression is feasible in E.coli. Hamartin's interaction region of tuberin is predicted to possess a potential leucine zipper region (81-121 aa) and a putative coiled-coil domain (346-371 aa). Recent studies have structurally proven the presence of a new component to the Tuberous sclerosis protein complex, which is known as TBC1D7. Many studies have been made with Tsc mutated cohorts and the researchers have tried to analyze the etiology of the disease biochemically. Yet, deciphering the structure of this tumor suppressor complex would not only satiate the search for answers in mTOR pathway but also throw limelight on the plethora of protein-protein interactions in various other significant pathways. Knowing the structure would enable us to envisage the design of agonist for these proteins to enhance their tumour suppressor activity.

1. Van Slegtenhorst, M. et al. (1998). Hum. Mol. Genet. 7, 1053-1057.

2. Li, Y. et al. (2004). Trends in Biochemical Sciences 29(1), 32-38.

3. Qin, J. et al. (2016). J. Biol. Chem. 291(16), 8591-8601.
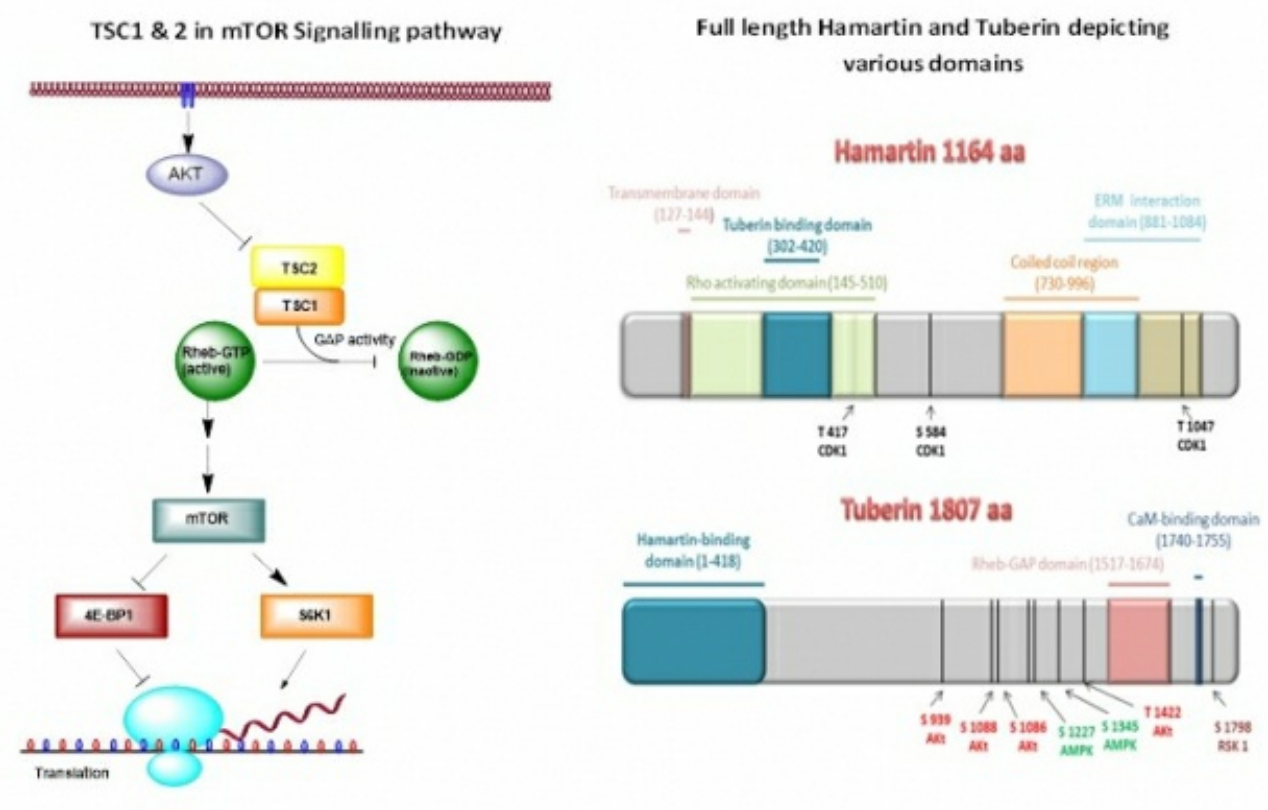

Keywords: Hamartin, Tuberin, Protein-protein interactions 